\title{
Perfiles genéticos bacterianos y análisis de brotes de las Enfermedades Transmitidas por Alimentos empleando Electroforesis de Campo Pulsado como herramienta para la vigilancia epidemiológica molecular
}

\author{
*Natalie Weiler, Flavia Ortiz, Maria Orrego, Claudia Huber, Mercedes Álvarez \\ Ministerio de Salud Pública y Bienestar Social. Laboratorio Central de Salud Pública. Departamento de Bacteriología \\ y Micología. Sección Enteropatógenos. Asunción, Paraguay \\ Cómo referenciar este artículo/ \\ How to reference this article: \\ Weiler N, Ortiz F, Orrego M, Huber C, Álvarez M. \\ Perfiles genéticos bacterianos y análisis de brotes de las \\ Enfermedades Transmitidas por Alimentos empleando \\ Electroforesis de Campo Pulsado como herramienta \\ para la vigilancia epidemiológica molecular. Mem. Inst. \\ Investig. Cienc. Salud. 2018; 16(2): 65-78
}

\section{R E S U M E N}

Las Enfermedades de Transmisión Alimentaria (ETA) son un problema de salud pública con altos índices de morbilidad y mortalidad a nivel global. La vigilancia y estudio de brotes de las ETA a través de Electroforesis de Campo Pulsado (PFGE) constituye un soporte fundamental para la investigación epidemiológica. El objetivo del estudio es presentar la base de datos de perfiles genéticos bacterianos y analizar brotes de enfermedades transmitidas por alimentos empleando Electroforesis de Campo Pulsado. Estudio descriptivo observacional de carácter retrospectivo, muestreo por conveniencia en el que fueron estudiados 778 aislamientos bacterianos causantes de ETA. La Base de Datos Nacional (BDN) quedó conformada por los siguientes patógenos entéricos; Salmonella spp., Shigella sonnei, Vibrio cholerae, Campylobacter spp., Escherichia coli O157:H7 y Escherichia coli no O157 caracterizados por una diversidad de patrones únicos, clusters y brotes. La BDN de Salmonella spp., quedó representada por un total de 558 cepas con 248 PUN, de las cuales $22,6 \%$ (126 cepas) corresponden a Salmonella enterica ser. Typhimurium, 20,6\% (115 cepas) a Salmonella enterica ser. Enteritidis, 9,1\% (51 cepas) a Salmonella enterica ser. Newport, 1,6\% (9 cepas) a Salmonella enterica ser. Muenchen, que al mismo tiempo son los serotipos que están asociados a brotes. Fueron confirmados un total de 13 brotes causados por Salmonella spp.; Shigella sonnei con 113 cepas estudiadas, 57 patrones únicos y 19 clusters detectados. Se identificaron 3 patrones PYJ16X01.0012, PYJ16X01.0034 y PYJ16X01.0014 como los predominantes. Vibrio cholerae con 18 cepas estudiadas, 9 patrones únicos y 4 clusters detectados. Se pudo establecer una relación genética del $100 \%$ entre cepas de Vibrio cholerae O1 biotipo El Tor serotipo Ogawa productora de toxinas ctxA y tcpA aislada del caso índice del brote de cólera. Campylobacter spp., con 62 cepas estudiadas, 42 patrones únicos y 10 clusters detectados. La BDN de $E$. coli productor de toxina shiga 0157 y no 0157, con 9 y 20 cepas de origen humano respectivamente, caracterizadas según sus factores de virulencia y subtipos. Se reconocieron 8 patrones electroforéticos PUN y 1 cluster para $E$. coli productor de toxina shiga O157, y 18 PUN y 1 clúster para $E$. coli productor de toxina shiga no O157.La disponibilidad de una Base de Datos Nacional de patógenos bacterianos transmitidos por alimentos constituye un importante avance para la salud pública, con un gran aporte en la vigilancia y epidemiología del país permitiendo la confirmación y detección de brotes discriminando aislamientos relacionados genéticamente y por consiguiente el estudio de relaciones clonales y probable origen.

Palabras clave: enfermedades transmitidas por alimentos, electroforesis en gel de campo pulsado, epidemiologia molecular. 


\title{
Bacterial genetic profiles and analysis of outbreaks of foodborne diseases using pulsed field gel electrophoresis as a tool for molecular epidemiological surveillance
}

\begin{abstract}
A B S T R A C T
Foodborne diseases (FBD) are a problem of public health with high indexes of morbidity and mortality at global level. The surveillance and study of outbreaks of the FBD through pulsed field gel electrophoresis (PFGE) is a fundamental support for epidemiological research. The aim of the study is to present the database of bacterial genetic profiles and analyze outbreaks of FBD using PFGE. This was an observational descriptive retrospective study with convenience sampling in which 778 bacterial isolates causing FBD were studied. The National Database (NDB) was made up of the following enteric pathogens causing FBD: Salmonella spp., Shigella sonnei, Vibrio cholerae, Campylobacter spp., Escherichia coli O157: H7 and Escherichia coli no 0157. Each of them was characterized by a diversity of unique patterns, clusters and outbreaks. The NDB of Salmonella spp. was represented by a total of 558 strains with 248 PUN, of which 22.6\% (126 strains) correspond to Salmonella enterica ser. Typhimurium, 20.6\% (115 strains) to Salmonella enterica ser. Enteritidis, $9.1 \%$ (51 strains) to Salmonella enterica ser. Newport, $1.6 \%$ (9 strains) to Salmonella enterica ser. Muenchen, which at the same time are the serotypes associated with outbreaks. A total of thirteen outbreaks caused by Salmonella spp., Shigella sonnei with 113 strains studied, 57 unique patterns and 19 clusters detected were confirmed. Three patterns PYJ16X01.0012, PYJ16X01.0034 and PYJ16X01.0014 were identified as the predominant. Vibrio cholerae with 18 strains studied, 9 unique patterns and 4 clusters were detected. A genetic relationship of $100 \%$ was established between strains of Vibrio cholerae O1 biotype El Tor serotype Ogawa toxin producer ctxA and tcpA isolated from the index case of the cholera outbreak. Campylobacter spp., with 62 strains studied, 42 unique patterns and 10 clusters were detected. The NDB of 0157 and non-O157 Shiga toxin producing E. coli 0157, with 9 and 20 strains of human origin respectively, were characterized according to their virulence factors and subtypes. We recognized 8 PUN electrophoretic patterns and 1 cluster for 0157 Shiga toxin producing E. coli, and 18 PUN and 1 cluster for non-0157 Shiga toxin producing E. coli. The availability of a National Database of bacterial pathogens transmitted by food constitutes an important advance for public health, with a great contribution to the surveillance and epidemiology of the country allowing the confirmation and detection of outbreaks discriminating genetically related isolates and therefore, the study of clonal relationships and probable origin.
\end{abstract}

Keywords: foodborne diseases, pulsed field gel electrophoresis, molecular epidemiology.

\section{INTRODUCCION}

Las Enfermedades de Transmisión Alimentaria (ETA) constituyen un problema de salud pública con altos índices de morbilidad y mortalidad a nivel global; afecta a países desarrollados y en mayor medida a países en vías de desarrollo ${ }^{(1)}$.

Según la Organización Mundial de la Salud (OMS), la incidencia anual de diarrea estimada en el mundo es de 1.500 millones de casos, con una mortalidad anual de 3 millones de niños menores de 5 años de edad. El $70 \%$ de las diarreas se originan por la ingestión de alimentos contaminados con microorganismos y/o sus toxinas ${ }^{(2,3)}$. Se han descrito alrededor de 250 agentes causantes de enfermedades transmitidas por alimentos (ETA), entre los que se incluyen bacterias, virus, hongos, parásitos, priones, toxinas y metales. En Estados Unidos de Norteamérica (E.U.A) se estima en 76 millones los casos anuales de ETA, lo que implica 325.000 hospitalizaciones y 5.000 muertes, lo cual representa costos significativos dentro de los gastos en salud ${ }^{(4)}$.

Centers for Disease Control (CDC), Food and Drug Administration (FDA) y el Departamento de Agricultura (USDA), indican como patógenos bacterianos de prevalencia en las ETA a Campylobacter spp., Listeria monocytogenes, Salmonella spp., Shigella spp., E. coli 0157, E. coli no-0157, y Yersinia spp. ${ }^{(5)}$. La contaminación de los alimentos puede ocurrir antes de su consumo, durante la elaboración, producción, distribución o comercialización; así mismo el comercio internacional creciente y el tránsito aéreo de personas son factores que contribuyen a la propagación de éstas enfermedades acortando distancias y tiempo de diseminación de las mismas ${ }^{(6)}$. 
La vigilancia molecular de los patógenos causantes de las ETA y estudio de brotes de origen alimentario a través de Electroforesis de Campo Pulsado (PFGE) constituye un soporte fundamental para la investigación epidemiológica, ya que utiliza una técnica molecular muy sensible y de alto poder de discriminación para conocer la relación genética entre aislamientos, incluso aquellos de casos esporádicos sin nexo epidemiológico evidente; además permite identificar fuentes o reservorios y contribuir a determinar vías de transmisión. Los perfiles genéticos permiten la creación de bases de datos con los patrones de bandas de bacterias que circulan en cada país construyendo de esta manera la Base de Datos Nacional (BDN). A su vez los países remiten a los laboratorios regionales los perfiles genéticos representantes de cada tipo de patrón denominado patrón único (PUN) que conforman la Base de Datos Regional (BDR) ${ }^{(7)}$.

Para garantizar la salud de la población es necesaria la aplicación de sistemas de control y asistencia eficaces en materia de inocuidad de alimentos, en ese aspecto el Laboratorio Central de Salud Pública (LCSP) como laboratorio de referencia para diagnóstico microbiológico y confirmación de patógenos causantes de ETA, en forma conjunta con la Dirección de Vigilancia Sanitaria (DGVS) y el Instituto Nacional de Alimentación y Nutrición (INAN), realiza un trabajo interdisciplinario para la vigilancia de enfermedades transmitidas por los alimentos y detección de brotes. La vigilancia molecular regional de patógenos entéricos en la Red PulseNet de América Latina y el Caribe se realiza a partir del 2004; Paraguay certifica en el 2011 y empieza la construcción de la base nacional. El objetivo de este trabajo es construir la base de datos de perfiles genéticos de bacterias y analizar brotes de las Enfermedades transmitidas por alimentos empleando Electroforesis de Campo Pulsado como herramienta para la vigilancia epidemiológica molecular.

Muñoz et al. $2006^{(8)}$ realizó un estudio de caracterización de Salmonella enterica ser. Typhimurium por PFGE, identificando patrones electroforéticos prevalentes, esto permitió conformar una base de datos nacional para la comparación de los perfiles en tiempo real, detectar brotes y relacionar casos aparentemente aislados. Rios et al $2009^{(9)}$, caracterizó por subtipificación molecular Salmonella enterica ser. Enteritidis, en muestras de diferentes orígenes, encontrando un alto predominio de fagotipo, reconociendo la utilidad de PFGE en la vigilancia. Diaz et al. $2014^{(10)}$, demostró por PFGE la relación genética de cepas de Salmonella enterica ser. Typhimurium en un brote de ETA. Campos J et al. 2011(11), en el primer informe de la red PulseNet AL y C, mostró la aplicación de PFGE para la comparación de aislados de Salmonella spp., y brotes en seis países diferentes de América Latina analizando la diversidad genética en especies de Salmonella spp., Zamudio et al. 2011 ${ }^{(12)}$ demostró la utilidad de PFGE en la vigilancia epidemiológica de ETA en Perú, describiendo un brote de Vibrio parahaemolyticus con aislamientos de casos clínicos, y presentó un caso de Vibrio cholerae con nuevo perfil genético en la región y la diseminación clonal de cepas de Salmonella enterica ser. Infantis; concluyendo que la aplicación de la técnica facilita la investigación epidemiológica de brotes. El estudio de Vélez $\mathrm{N}$ et al. $2015^{(13)}$ determinó la relación genotípica de aislamientos de $S$. sonnei identificando patrones para la base de datos a nivel nacional mediante PFGE. Ruiz et al. $2014^{(14)}$, caracterizó cepas de Campylobacter coli aislados de muestras clínicas, reconociendo varios clusters entre las cepas estudiadas. Kalender et al. $2016^{(15)}$, realizó la caracterización de Escherichia coli 0157:H7 de cepas aisladas de heces de res por varios métodos moleculares incluyendo PFGE, destacó la diversidad genética de las cepas en estudio con varios patrones únicos y clusters detectados. Oderis et al. $2018^{(16)}$, caracterizó cepas de Escherichia coli productor de toxina shiga de muestras pediátricas detectando patrones con al menos un $88 \%$ de similitud y varios clusters que agruparon cepas con perfiles idénticos. El objetivo de estudio fue presentar la base de datos de perfiles genéticos bacterianos y analizar brotes de enfermedades transmitidas por alimentos empleando Electroforesis de Campo Pulsado.

\section{MATERIALES Y METODOS}

Estudio descriptivo observacional de carácter retrospectivo con un muestreo por conveniencia con 778 aislamientos bacterianos de patógenos entéricos causantes de ETA recuperados a partir de muestras diarreicas de origen humano, muestras de origen alimentario, animal y medio ambiente, desde el 2008 al 2017; remitidos al Laboratorio Central de Salud Pública por distintas instituciones de salud y otras pertenecientes a la Red de Vigilancia de Enteropatógenos del país. Fueron seleccionadas aquellas cepas de patógenos entéricos provenientes de brotes; cepas de patógenos entéricos portadoras de 
multirresistencia a antibióticos; cepas de patógenos entéricos con un aumento inesperado en cuanto a prevalencia; cepas de patógenos entéricos de diferentes especies y/o serotipos.

Las cepas incluidas en el estudio conservadas a $-80^{\circ} \mathrm{C}$, fueron subcultivadas en agar nutritivo y sometidas a la técnica de PFGE según protocolos estandarizados de la Red PulseNet ${ }^{(17)}$ y como cepa standard Salmonella enterica ser. Braenderup $\mathrm{H}-9812^{(18)}$. Se utilizó equipo CHEFF-DR III System Biorad de procedencia U.S.A. Los patrones electroforéticos obtenidos, fueron analizados con el software Gel Compare II versión 5.1 (Applied Maths) procedencia Bélgica, aplicando el coeficiente de Dice y el método UPGMA, con $1,5 \%$ de tolerancia y $1,5 \%$ de parámetros de optimización para el análisis habitual, según criterios recomendados por PulseNet. Los datos se tabularon y analizaron en el programa Microsoft Excel ${ }^{\mathrm{TM}}$ para obtención de porcentajes.

Los distintos patrones electroforéticos fueron nombrados utilizando la nomenclatura siguiendo las pautas de PulseNet LA \& C, que incluye 2 letras para el país o región, 3 para el serotipo, 3 caracteres para la enzima y 4 dígitos para el número de perfil (por ejemplo, ALJPPX01.0001) ${ }^{(19)}$ siendo considerados como patrones únicos nacionales (PUN) a aquellos perfiles genéticos específicos de una especie bacteriana que permite la subtipificación de las mismas y son representantes en la BDN. Además se procedió a enviar estos PUN a la BDR de manera a conformar una base internacional que permita realizar comparaciones entre perfiles de diferentes países.

Para el análisis de brotes se identificó en cada caso el probable patrón del brote, comparándose con los demás patrones de la base de datos. Patrones que son claramente diferentes del patrón de brote (menos de $50 \%$ de fragmentos en común) se consideraron no relacionados. Patrones que presentaron diferencias del patrón de brote por dos o tres diferencias de fragmentos se consideraron subtipos del patrón de brote según tabla de comparación de perfiles electroforéticos Tenover ${ }^{(20)}$.

\section{RESULTADOS}

La Base de Datos Nacional (BDN) quedó conformada por los siguientes patógenos entéricos causantes de ETA como Salmonella spp., Shigella sonnei, Vibrio cholerae, Campylobacter spp., Escherichia coli 0157:H7 y Escherichia coli no 0157, cada una de ellas caracterizadas por una diversidad de patrones únicos, clusters y brotes (Tabla 1).

Tabla 1. Base de Datos Nacional de Paraguay. Distribución de patógenos entéricos analizadas por PFGE

\begin{tabular}{lllll}
\hline $\begin{array}{l}\text { Cepa bacteriana } \\
\text { entérica }\end{array}$ & $\begin{array}{l}\mathbf{N}^{\circ} \text { cepas } \\
\text { estudiadas }\end{array}$ & $\begin{array}{l}\mathbf{N}^{\circ} \text { de } \\
\text { patrones (PUN) }\end{array}$ & $\begin{array}{l}\mathbf{N}^{\circ} \\
\text { clusters }\end{array}$ & $\begin{array}{l}\mathbf{N}^{\circ} \text { brotes } \\
\text { confirmados }\end{array}$ \\
\hline Salmonella spp. & 558 & 248 & 72 & 13 \\
Shigella sonnei & 113 & 57 & 19 & 0 \\
Vibrio cholerae & 18 & 9 & 4 & 1 \\
E coli O157:H7 & 9 & 8 & 1 & 0 \\
E coli no O157:H7 & 18 & 14 & 1 & 0 \\
Campylobacter spp. & 62 & 42 & 10 & 0 \\
\hline
\end{tabular}

\section{Salmonella spp.}

La BDN de Salmonella spp., quedó representada por un total de 558 cepas con 248 PUN, de las cuales $22,6 \%$ (126 cepas) corresponden a Salmonella enterica ser. Typhimurium, $20,6 \%$ (115 cepas) a Salmonella enterica ser. Enteritidis, 9,1\% (51 cepas) a Salmonella enterica ser. Newport, 1,6\% (9 cepas) a Salmonella enterica ser. Muenchen, que al mismo tiempo son los serotipos que están asociados a brotes; el 46,1\% (257 cepas) restantes corresponden a otros serotipos. Fueron confirmados un total de 13 brotes causados por Salmonella spp; 7 brotes relacionados con Salmonella enterica ser. Enteritidis, entre los cuales se destacan el brote ocurrido en un patio de comidas en la ciudad de Fernando de la Mora en el 2012 con un total de 33 personas afectadas, de los cuales 23 fueron hospitalizados y un segundo brote en el mismo año ocurrido en un albergue de niños con un total de 23 niños afectados. En ambos casos se pudo establecer una relación entre el alimento ingerido y los pacientes (Figuras $1 \mathrm{~A}$ y $\mathrm{B}$ ), otro de los serotipos detectados fue 
Salmonella enterica ser. Newport en 3 brotes (Figuras 1D y E), Salmonella enterica ser. Muenchen (Figura 1F) y Salmonella enterica ser. Typhimurium en 1 y 2 brotes respectivamente (Figuras $1 \mathrm{G} \mathrm{y} \mathrm{H}$ ).

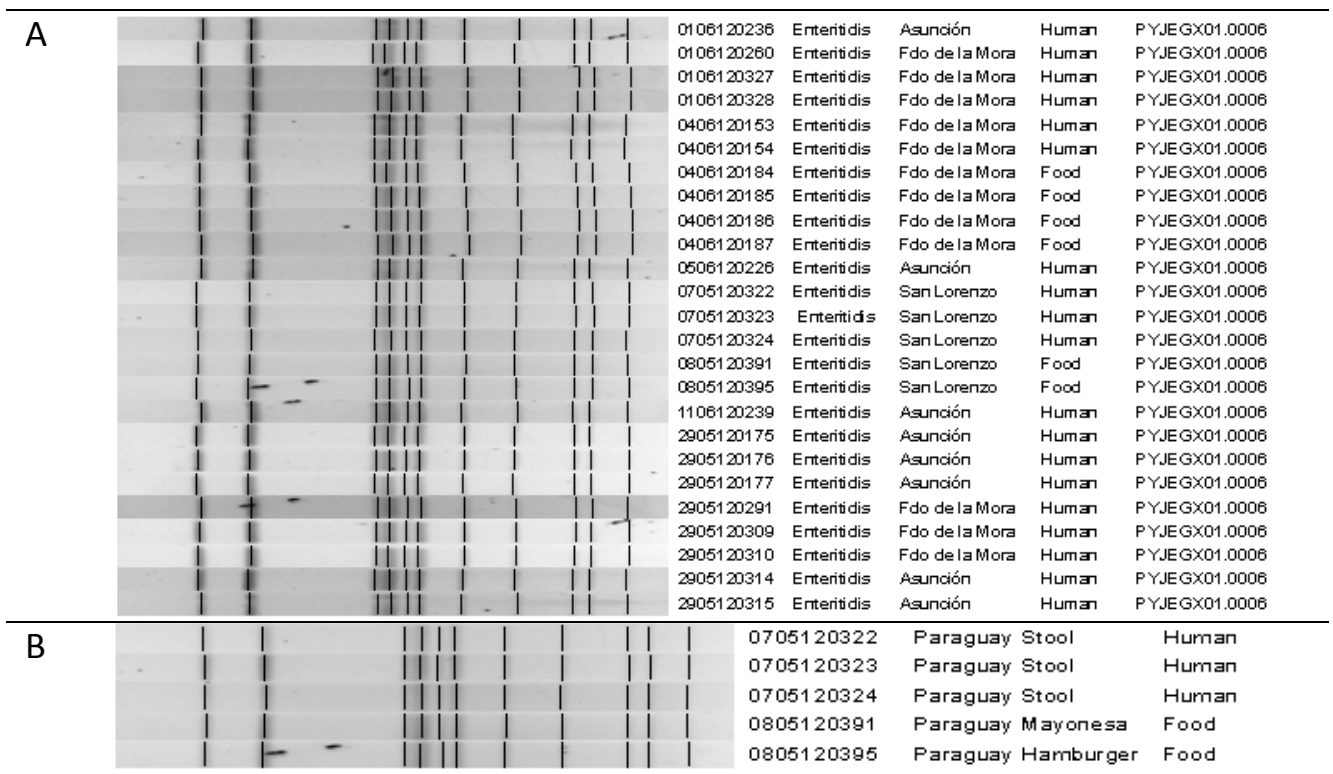

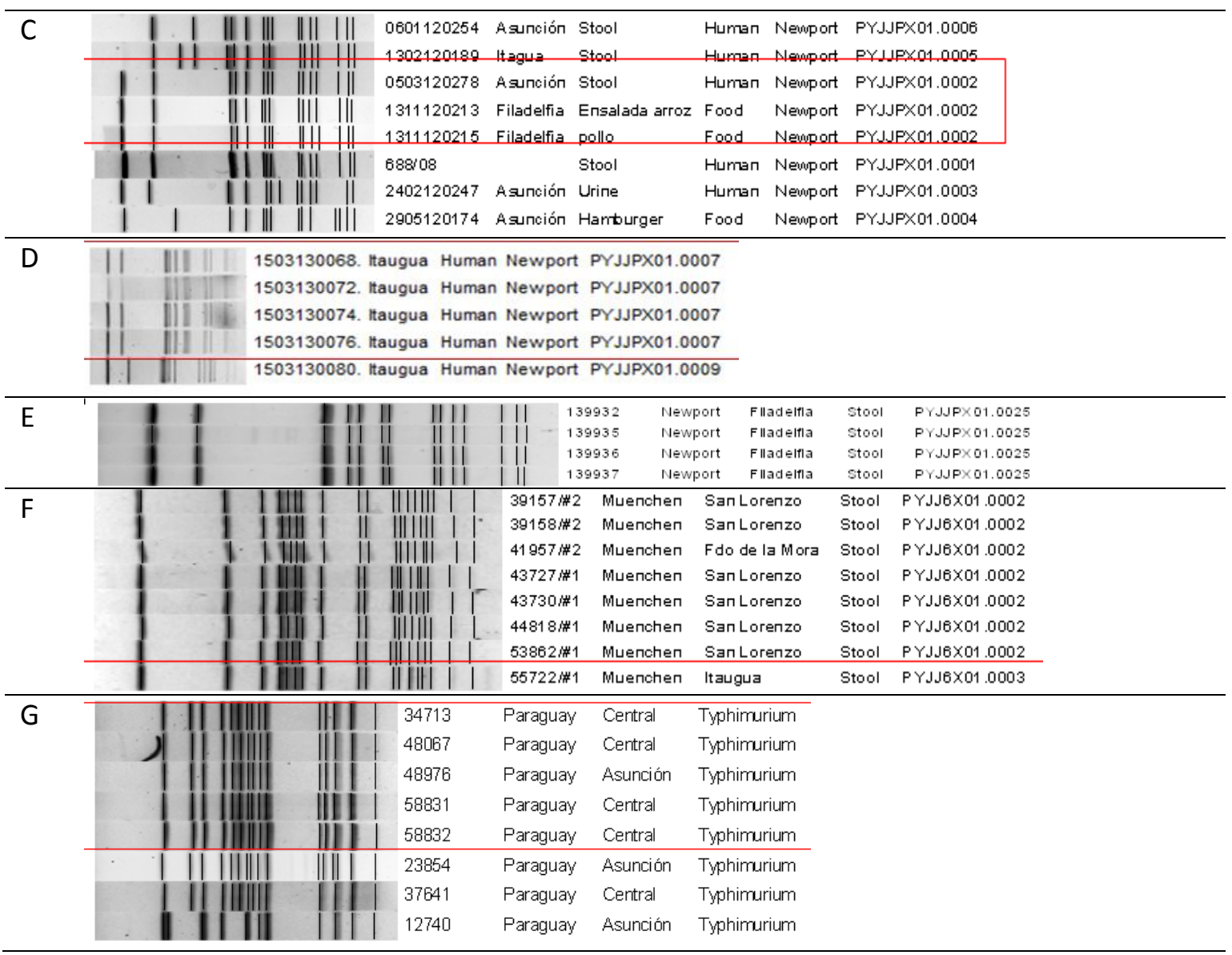




\begin{tabular}{lllllllllll}
\hline$H$ & \\
\hline$H$
\end{tabular}

Figura 1. A. Brote -Patio de Comida-Fernando de Fernando de la Mora/2012. Patrones electroforéticos PFGE-XbaI de Salmonella enterica ser. Enteritidis de un brote alimentario. Comparación de muestras de pacientes, manipuladores y alimentos. Alimento implicado: sandwich de jamón y queso, sandwich de verduras, empanadas de pollo y croqueta. Se observa una relación genética del $100 \%$ entre todas las cepas. B: Brote de S. enterica ser. Enteritidis San Lorenzo/2012 en comedor para niños con 23 niños afectados y 4 internaciones. Alimento implicado: hamburguesa de carne y mayonesa. Relación genética del $100 \%$ entre paciente y alimento implicado. C: Brote de S. enterica ser. Newport -Filadelfia/2012 en evento vecinal con 38 personas; 2 hospitalizadas. Alimento implicado: ensalada de arroz con pollo. Asociación entre muestra de un paciente y alimento implicado. D: Brote de $S$. enterica ser. Newport intrahospitalario en 4 personas de Clínica Médica, UCI y Nefrología del mismo hospital/2013. E: Brote alimentario de Salmonella enterica ser. Newport en Filadelfia-Boquerón/2016. F: Brote intrahospitalario de $S$. enterica ser. Muenchen en 2 hospitales en diferentes salas/2015. G: Brote de $S$. enterica ser. Typhimurium BLEE. 2015. Cepas remitidas de diferentes instituciones de salud. H: Brote alimentario de S. enterica ser. Typhimurium /2017. Alimento implicado: Carne asada, mayonesa.

\section{Shigella sonnei}

La BDN de Shigella sonnei quedó representada por un total de 113 cepas analizadas, todas de origen humano. La distribución de patrones electroforéticos de este patógeno entérico presentó una alta variabilidad genética con 57 patrones únicos agrupados en varios clusters que fueron detectados de casos esporádicos sin nexo epidemiológico aparente. Se identificaron 3 patrones PYJ16X01.0012, PYJ16X01.0034 y PYJ16X01.0014 como los predominantes (Figura 2A). Estas cepas fueron seleccionadas debido a un aumento de prevalencia respecto a $S$. flexneri. Así mismo se analizaron cepas con portación de mecanismos de resistencia a antimicrobianos desde el año 2017, fenómeno ya descripto a nivel regional y mundial; en 22 cepas de $S$. sonnei con mecanismos de resistencia a cefalosporinas y a quinolonas se observó escasa variabilidad genética destacándose la presencia de 2 grupos clonales estrechamente relacionados provenientes de centros hospitalarios con diversa ubicación geográfica lo que sugiere la transmisión de estas cepas entre los distintos nosocomios. (Figura 2B). 


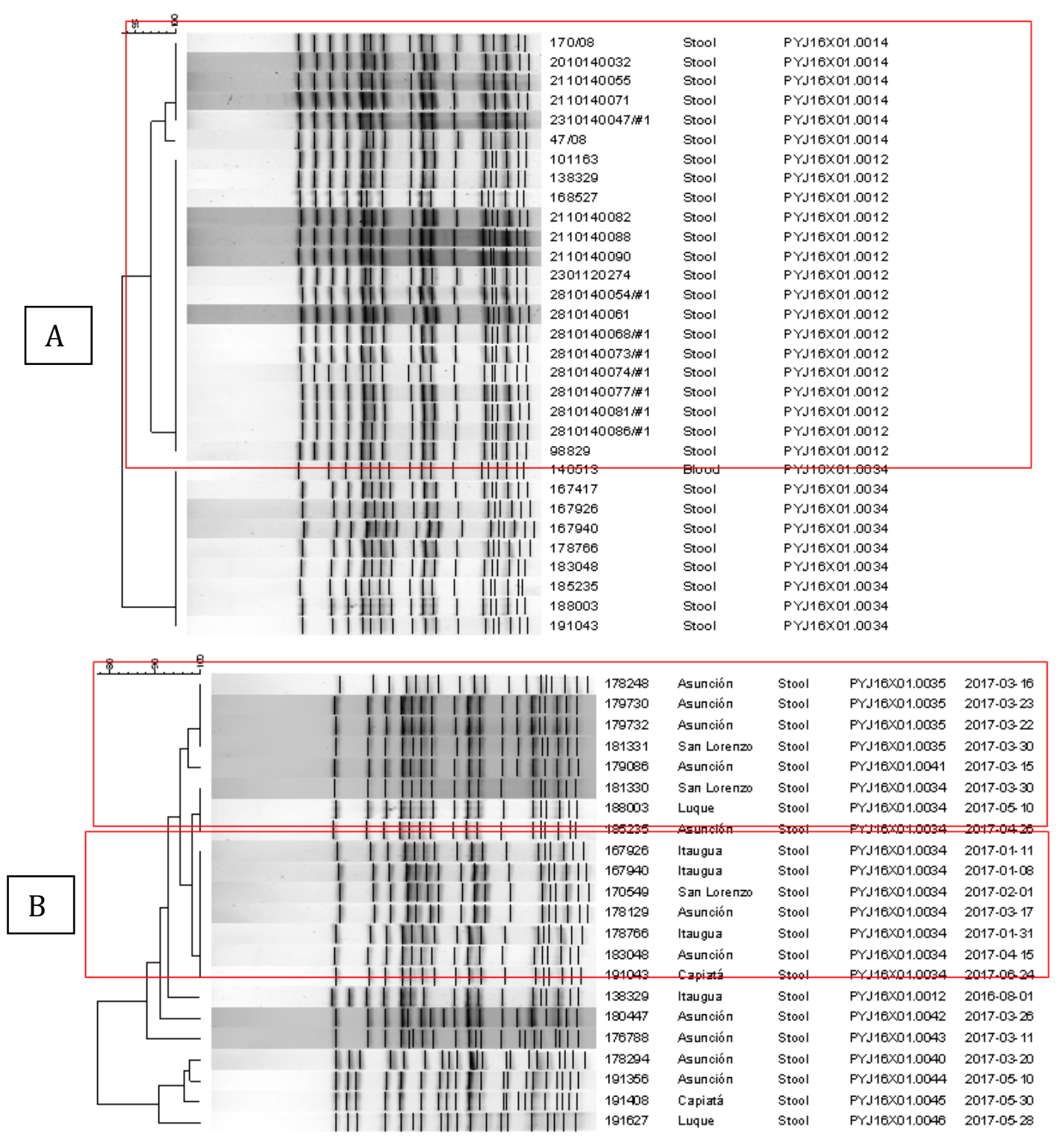

Figura 2. A. Clúster de PUN prevalentes de Shigella sonnei. Enzima de restricción XbaI. 2010-2017. Paraguay. S. sonnei posee alta diversidad de patrones electroforéticos en la base de datos nacional. Se puede apreciar 3 clúster con los patrones (PUN) más frecuentes PYJ16X01.0012, PYJ16X01.0034 y PYJ16X01.0014. B. S. sonnei con resistencia adquirida ctx-M y qnr-S - 2017. El análisis de los fragmentos de restricción evidencia que las cepas analizadas están genéticamente relacionadas con dos grupos clonales circulantes entre diferentes centros hospitalarios.

\section{Vibrio cholerae}

La BDN de Vibrio cholerae quedó representada por 18 cepas que fueron analizadas por PFGE con 9 PUN (enzimas de restricción SfiI y NotI) y 4 clusters. Las mismas corresponden a aislamientos de muestras humanas y ambientales recolectadas en el año 2009 de reservorios de agua y de casos de diarrea en el marco de una investigación de brote en el departamento de Boquerón (Chaco Paraguayo). Se pudo establecer una relación genética del $100 \%$ entre la cepa de Vibrio cholerae O1 biotipo El Tor serotipo Ogawa productora de toxinas ctxA y tcpA aislada del caso índice del brote de cólera ocurrido en el año 2009 en Filadelfia-Boquerón y la cepa aislada de agua de tajamar que se presumió ser el reservorio (Figura $3 \mathrm{~A}$ ). Además se pudo establecer una estrecha relación entre las cepas del brote de Filadelfia y una cepa aislada en el Chaco Argentino en el año 2005 y establecer diferencias significativas con las cepas de la epidemia de los años 90 (Figura 3B). 


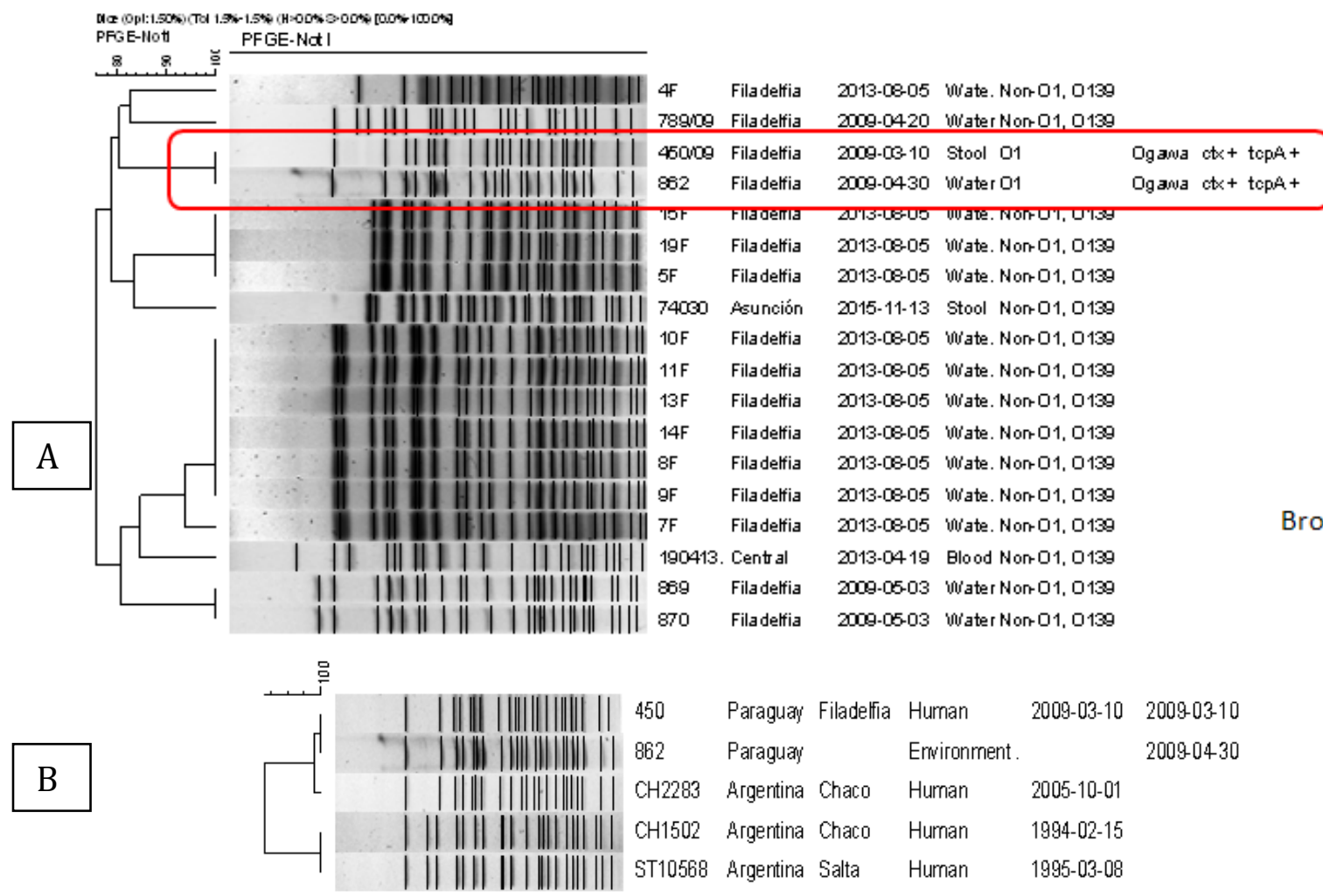

Brote 2009

Figura 4. A: Distribución de patrones electroforéticos de Vibrio cholerae. Enzima de restricción SfiI. 2010-2017. Paraguay. Se observa un cluster 4 grupos clonales muy conservados, entre ellas 1 grupo clonal correspondiente al brote de Filadelfia Chaco causado por Vibrio cholerae serogrupo 01 serotipo Ogawa portador de con genes de virulencia ctx y tcpA. Las demás cepas corresponden a cepas humanas y ambientales no epidémicas. B: Relación genética entre los aislamientos recuperados en Paraguay durante el brote del 2009 y el aislamiento recuperado en Argentina en 2005.

\section{Campylobacter jejuni}

La Base de Datos Nacional de Campylobacter jejuni, quedó representada por 62 cepas analizadas por PFGE con la enzima SmaI, 54.8\% (34 cepas) corresponden a cepas de origen humano, $12.9 \%$ ( 8 cepas) a cepas de alimentos y $32.3 \%$ (20 cepas) de animales (Figura 4 ) recolectadas en el mismo periodo de tiempo. Se observó una alta diversidad genética con 42 PUN; y 10 clusters, $40 \%$ (4 clusters) entre cepas de origen humanos, $30 \%$ (3 clusters) entre cepas animales, $10 \%$ ( 1 cluster) entre cepas de alimentos y $20 \%$ ( 2 clusters) con una relación genética del $100 \%$ entre cepas de origen humano y animal (Figura 4). 


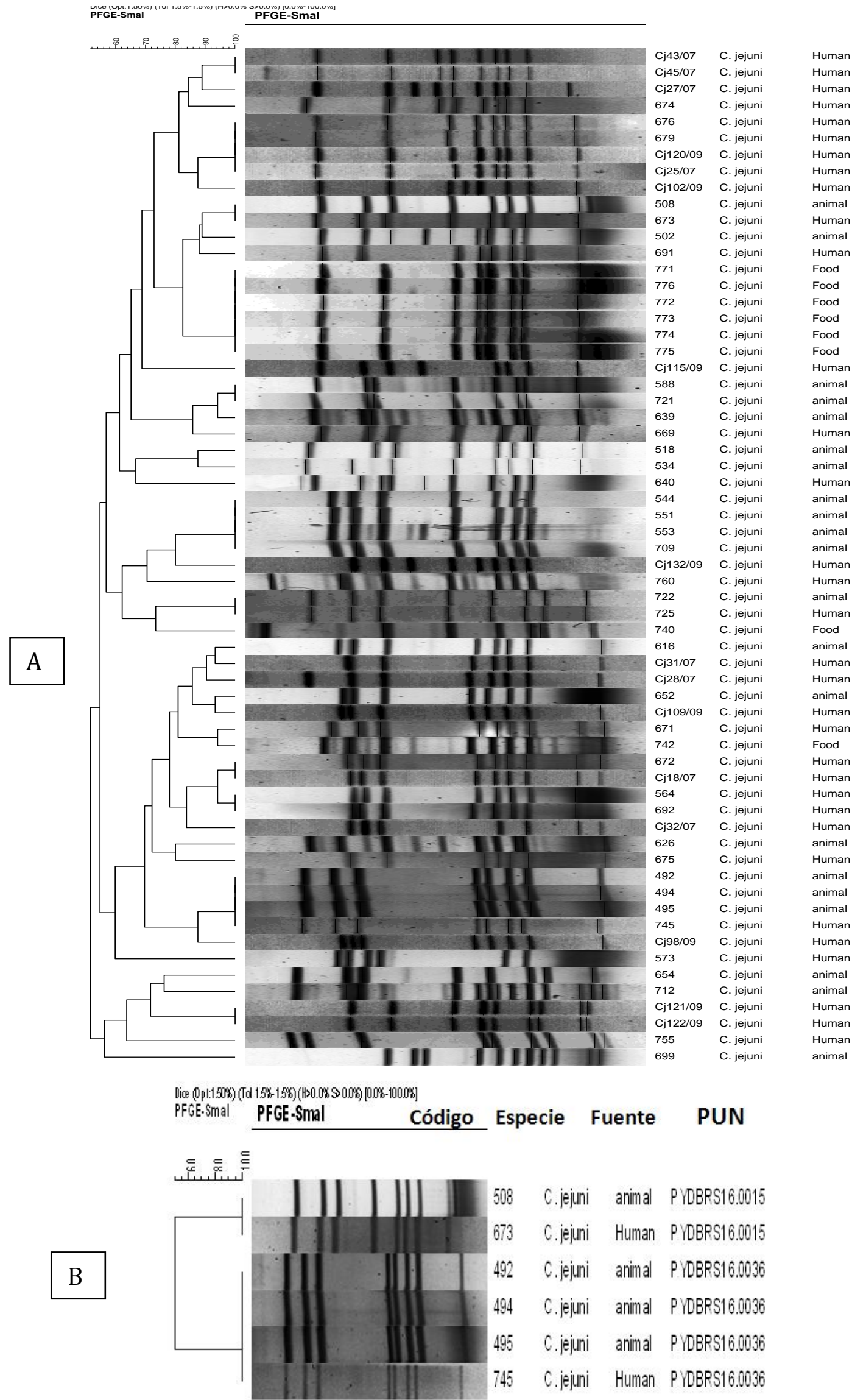

Figura 4. A. Distribución de patrones electroforéticos de C. jejuni. Enzima de restricción SmaI. 2008-2017. Paraguay. B: Clusters de $C$. jejuni en animales y humanos como parte de la Vigilancia integrada de la resistencia. 


\section{E. coli productor de toxina shiga 0157 y no 0157.}

La BDN de $E$. coli productor de toxina shiga 0157 y no 0157 , quedó conformada por 9 y 20 cepas de origen humano respectivamente, caracterizadas según sus factores de virulencia y subtipos. Estas fueron analizadas por PFGE con la enzima XbaI reconociendo 8 patrones electroforéticos PUN y 1 cluster para $E$. coli productor de toxina shiga 0157 (Figura 14) y 18 PUN y 1 clúster para E. coli productor de toxina shiga no O157. (Figura 5).
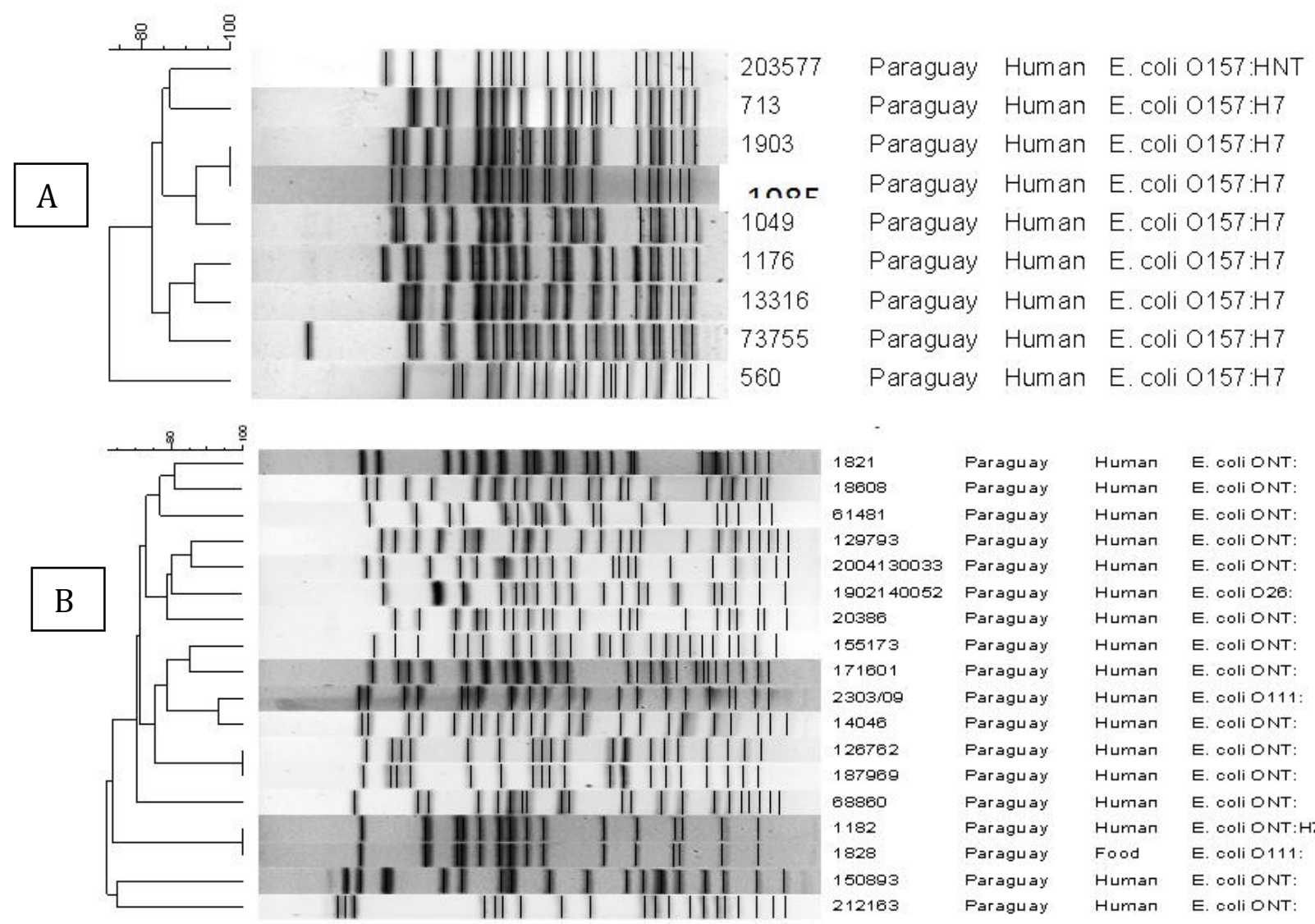

$$
\begin{array}{lll}
\text { Paraguay } & \text { Human } & \text { E. coli ONT: } \\
\text { Paraguay } & \text { Human } & \text { E. coli ONT: } \\
\text { Paraguay } & \text { Human } & \text { E. coli ONT: } \\
\text { Paraguay } & \text { Human } & \text { E. coli ONT: } \\
\text { Paraguay } & \text { Human } & \text { E. coli ONT: } \\
\text { Paraguay } & \text { Human } & \text { E. coli O26: } \\
\text { Paraguay } & \text { Human } & \text { E. coli ONT: } \\
\text { Paraguay } & \text { Human } & \text { E. coli ONT: } \\
\text { Paraguay } & \text { Human } & \text { E. coli ONT: } \\
\text { Paraguay } & \text { Human } & \text { E. coli O111: } \\
\text { Paraguay } & \text { Human } & \text { E. coli ONT: } \\
\text { Paraguay } & \text { Human } & \text { E. coli ONT: } \\
\text { Paraguay } & \text { Human } & \text { E. coli ONT: } \\
\text { Paraguay } & \text { Human } & \text { E. coli ONT: } \\
\text { Paraguay } & \text { Human } & \text { E. coli ONT:H7 } \\
\text { Paraguay } & \text { Food } & \text { E. coli O111: } \\
\text { Paraguay } & \text { Human } & \text { E. coli ONT: } \\
\text { Paraguay } & \text { Human } & \text { E. coli ONT: }
\end{array}
$$

Figura 5. Distribución de patrones electroforéticos y enzima de restricción XbaI A. de E. coli 0157. B. de E. coli no 0157 .

\section{DISCUSIÓN}

La base de datos nacional contiene 778 cepas analizadas, cuyos perfiles electroforéticos permiten realizar comparaciones arrojando porcentajes de similitud e identificando clusters o cepas con alta relación genética y de esta manera contribuir a la resolución de brotes.

La BDN de Salmonella spp contiene actualmente 44 serotipos con perfiles diferentes utilizando la enzima XbaI. En este trabajo presentamos la diversidad de los subtipos genéticos encontrados en las serovariedades más frecuentes así como información sobre brotes. Se destaca una alta representatividad en términos temporales y geográficos y variabilidad genética de este patógeno, lo que nos permite realizar comparaciones de perfiles aumentando la probabilidad de resolución. Este patógeno es el más relacionado a brotes de ETA y $S$. Enteritidis entre los serotipos con mayor presentación de casos de brotes. En el brote ocurrido en el 2012, en el cual estuvieron implicadas personas con síndrome diarreico agudo que ingirieron alimentos provenientes de un supermercado, se aisló S. Enteritidis de las muestras de origen humano tanto de pacientes como de manipuladores de alimentos y de las muestras de alimentos consumidos. La subtipificacion molecular de las cepas aisladas mostró un $100 \%$ de relación genética lo que permitió establecer una probable fuente de origen. El amplio uso de PFGE en la subtipificación, ha demostrado que este agente infeccioso tiene un alto grado de estabilidad genética, lo que concuerda con los resultados obtenidos en nuestro país, donde la mayoría de los subtipos está estrechamente relacionado y son similares a los que se han reportado en otros lugares del mundo Ríos et al. $2009^{(9)}$. Esta clonalidad entre las cepas, es característica limitante en cuanto a la capacidad de discriminación de las mismas, por lo que es necesaria la 
implementación de nuevas técnicas moleculares que posean un mayor grado de discriminación. Otro brote que se destaca en el mismo año con el mismo serotipo, se desarrolla en un comedor para niños (23 niños afectados de los cuales, 4 niños estuvieron internados), se determinó como alimento implicado hamburguesa y mayonesa utilizada como condimento.

A fines del 2012, se reporta un probable brote alimentario en Filadelfia-Chaco, con 38 personas intoxicadas y 2 personas hospitalizadas en un evento vecinal. Se recuperando 1 muestra de origen humano y los alimentos implicados (ensalada de arroz y pollo), de las cuales se aisló $S$. Newport. El perfil electroforético de las cepas fue del $100 \%$ de similitud. Al mismo tiempo, se compararon con otros perfiles de la BDN, buscando perfiles similares, al no encontrar similitud, se designó un nuevo PUN a las 3 cepas implicadas. En el año 2013, se reporta en una institución de salud el aislamiento de Salmonella sp., de pacientes internados de distintos servicios como el de clínica médica, unidades de cuidados intensivos y nefrología. Se analizaron 4 cepas remitidas al LCSP, identificadas fenotípicamente como $S$. Newport con un $100 \%$ de similitud entre los perfiles electroforéticos. En el 2016, en Filadelfia, Chaco, se presentó un aumento de aislamientos de $S$. Newport todas provenientes de muestras diarreicas de pacientes que se presentaron al Hospital en un periodo de diferencia de dos días, no se conocen datos epidemiológicos sobre el origen común de los pacientes o si hubo algún alimento implicado reconocido en el mismo. Las cepas analizadas por PFGE dieron un $100 \%$ de relación genética y dando lugar a un cluster nuevo no identificado en la BDN.

En el 2015, se reportan en hospitales del departamento central varios aislamientos de $S$. Muenchen con multirresistencia antimicrobiana captados en un periodo de un mes. La comparación de perfiles electroforéticos de las cepas implicadas presenta un $100 \%$ de relación genética, concluyendo un probable brote intrahospitalario. Esto se informó a la DGVS, para su intervención a través del Departamento de control de infecciones de cada hospital implicado y tomar las medidas pertinentes a cada caso.

Actualmente $S$. Typhimurium está posicionada como la serovariedad de mayor prevalencia, dejando atrás a S. Enteritidis coincidiendo con Diaz et al. $2014^{(10)}$ y Campos J. et al. 2011 11 quienes observan un incremento a nivel mundial. La multirresistencia observada en este serotipo, se refiere a varios mecanismos de resistencia como bombas de eflujo para antimicrobianos como tetraciclina, la resistencia a Beta-lactámicos por inactivación del antibiótico por Betalactamasas, modificación del sitio de unión de las proteínas fijadoras de penicilina entre otros. También es importante destacar otros de los factores que influyen sobre la resistencia como el amplio uso de antibióticos en los animales, los cuales actúan como reservorios que posteriormente son utilizados como fuente de alimento para el consumo humano.

En los meses de abril a agosto del año 2015, se reportan desde 4 instituciones de salud tanto del departamento central como capital, el aislamiento de $S$. Typhimurium con portación de mecanismos de resistencia BLEE. Con este criterio de portación de multirresistencia, se realizó la comparación de los perfiles electroforéticos de las cepas aisladas y otras presentes en la BDN, encontrando en 5 cepas remitidas de dichas instituciones un $100 \%$ de similitud de los perfiles electroforéticos. En el año 2017, se reporta un probable brote alimentario en una estancia donde el alimento implicado fue carne asada y mayonesa. Se recuperaron 20 muestras de origen humano, de las cuales en 14 de ellas se aisló S. Typhimurium con un $100 \%$ de similitud de perfiles electroforéticos.

Esta técnica también permitió además la generación de alertas, por detección de clusters, reportándose estas las autoridades pertinentes. Estas alertas fueron generadas principalmente por la detección de un inusitado número de aislamientos de Shigella sonnei que presentaron un aumento significativo en cuanto a su prevalencia respecto a Shigella flexneri, además de la portación de varios mecanismos de resistencia. Se estudiaron 113 cepas de Shigella sonnei por PFGE y se pudo demostrar la presencia de 2 grupos clonales en 22 cepas muy relacionadas genéticamente, que estaban diseminadas en diferentes centros hospitalarios. Si bien no se pudo confirmar la existencia de un brote esta alerta permitió la intervención de las autoridades responsables. Se logró la construcción de la base de datos nacional de Shigella sonnei, así como lo realizó Velez et al. $2015^{(13)}$.

El aislamiento de Vibrio cholerae a partir de muestras humanas es infrecuente en Paraguay. El estudio del brote de Vibrio cholerae en el 2009, por PFGE permitió confirmar una relación genética del $100 \%$ entre las cepas estudiadas. Este brote se produjo entre 
diciembre de 2008 a abril de 2009 en Filadefia-Boquerón (Chaco Paraguayo). Se recuperaron dos aislamientos; uno en una muestra de heces del caso índice y otro en una muestra de agua de tajamar, caracterizándose como Vibrio cholerae 01 biotipo El Tor serotipo Ogawa productora de toxinas ctxA y tcpA. Estas cepas pertenecientes al brote fueron comparadas con una cepa aislada en el Chaco argentino en el año 2005 y con cepas pertenecientes a la epidemia de cólera de los 90 encontradas en la base de datos regional, en donde, se pudo detectar relación genética con la cepas argentina del año 2005 no así con las cepas de brote de los 90. Así también Zamudio et al 2011 ${ }^{(12)}$ en su trabajo de investigación realizó la misma comparación con cepas de Argentina, Paraguay, y las cepas de la epidemia de cólera del año 1991 para buscar alguna relación genética con las cepas reportadas de Perú, sin encontrar asociación genética alguna con sugiriendo un origen distinto. Coincidimos con el estudio de Zamudio et al. $2011^{(12)}$ en la importancia de esta herramienta molecular en la investigación de brotes.

La base de datos nacional de Campylobacter spp., se encuentra constituida por cepas de origen animal, alimentario y humano; con una gran diversidad de perfiles genéticos detectados en estos tres grupos coincidiendo con Ruiz et al. 2014 ${ }^{(14)}$. Entre las cepas de origen humano hemos encontrado perfiles genéticos similares, así como también entre cepas de animales y entre cepas de alimentos. Las mismas fueron recolectadas y analizadas en un periodo de 12 meses. Así también se pudo detectar 2 clúster de $C$. jejuni entre humanos y animales, como parte de la vigilancia integrada, presentando un probable origen común.

En referencia a las cepas de E. coli 0157 y no 0157 analizadas por esta técnica, es importante destacar la baja prevalencia de estos patógenos entéricos en nuestro país ${ }^{(23)}$ y otros países como lo destaca Reyes et al. $2013^{(22)}$ con un $0.8 \%$ y $2.6 \%$ para 0157 y no 0157 respectivamente. Hemos detectado gran diversidad genética; lo que también lo destacan Kalender et al. $2016^{(15)}$ y Oderis et al. $2018^{(16)}$.

Con respecto a comparaciones con la BDR, se han realizado con los diferentes patógenos entéricos analizados de acuerdo a la aparición de brotes en nuestro país y a demanda de los demás países de la región en caso de brotes detectados a nivel internacional, un ejemplo de ello es la detección de clones similares entre las cepas del brote de Vibrio cholerae detectado en Paraguay en el año 2009 y la cepa de Argentina del año 2005.

Se destaca sin embargo que esta técnica posee sus limitaciones ya que es muy laboriosa y se necesita un mínimo de dos días para la realización de las corridas además de ser operador dependiente requiriendo una certificación por cada patógeno y ser influida por diferentes variables como ser calidad del agua, calidad de la enzima, la temperatura ambiental. Si bien el PFGE ha demostrado ser notablemente útil para detectar clusters carece de la capacidad discriminatoria y filogenética comparada con métodos más avanzados ya que las inserciones, deleciones, reordenamientos y mutaciones puntuales en el sitio de restricción pueden presentar a aislamientos altamente relacionados como genéticamente diferente y del mismo modo, aislamientos que no están altamente relacionados parecer indistinguibles por PFGE. Ambos escenarios pueden provocar errores que dificulten las investigaciones epidemiológicas.

Los resultados demuestran la importancia de esta técnica y de la disponibilidad de una Base de Datos Nacional para fortalecer la vigilancia de laboratorios tanto en su componente clínico-humano, epidemiológico y ambiental, permitiendo definir con mayor certeza el rol de los diversos subtipos genéticos, determinar los nexos entre casos y fuentes, hacer un adecuado seguimiento de los clones circulantes a nivel nacional generando alertas al sistema de salud. Al mismo tiempo contribuye a la detección y confirmación de brotes discriminando los aislamientos relacionados genéticamente y de un probable origen. Con este trabajo se presenta la consolidación de la construcción de la base genética nacional de patógenos de transmisión alimentaria y resaltamos como logro fundamental haber introducido el uso de esta técnica en forma rutinaria en las actividades del Laboratorio lo que ha permitido el fortalecimiento de la Vigilancia de las ETA en Paraguay.

\section{AGRADECIMIENTOS}

Agradecemos a Enrique Pérez, Isabel Chinen, coordinadores de la Red PulseNet ALyC Josefina Campos del Instituto Carlos G. Malbrán así como a los laboratorios de la Vigilancia de Enteropatógenos por el esfuerzo continuo para la recuperación de los aislamientos y el envío de cepas bacterianas. 


\section{REFERENCIAS BIBLIOGRÁFICAS}

1. Olea A, Díaz J, Fuentes $R$, Vaquero $A$, García M. Vigilancia de brotes de enfermedades transmitidas por alimentos en Chile. Rev. chil. infectol. 2012;29(5):504-10. Disponible en:https://scielo.conicyt.cl/scielo.

2. WHO. Consultation to develop a strategy to estimate the global burden of foodborne diseases. World Health Organization, Geneva, $\quad 2007$. http://www.who.int/

foodsafety/publications/foodborne_disease / burden_sept06/en/index.html.

3. Jones KE, Patel NG, Levy MA, Storeygard A, Balk D, Gittleman JL, et al. Global trends in emerging infectious diseases. Consortium for Conservation Medicine. USA. Nature 2008; 451: 990-3. Disponible en: https://www.nature.com > nature > letters.

4. Mead PS, Slutsker L, Dietz V, McCaig LF, Bresee JS, Shapiro $C$ et al. Food related illness and death in the United States. Emerg Infect Dis 1999; 5: 607-25. Disponible en: DOI: $10.3201 /$ eid0505.990502

5. Hoffmann S, Devleesschauwer B, Aspinall W, Cooke R, Corrigan T. Attribution of global foodborne disease to specific foods: Findings from a World Health Organization structured expert elicitation. PLoS ONE 12(9): e0183641. Disponible en: https://doi.org/10.1371/journal.

pone. 0183641

6. Kooper G, Calderón G, Schneider S, Domínguez W, Gutiérrez G. Enfermedades transmitidas por alimentos y su impacto económico. Estudio de caso en Costa Rica, El Salvador, Guatemala, Honduras y Nicaragua. ORGANIZACIÓN DE LAS NACIONES UNIDAS PARA LA AGRICULTURA Y LA ALIMENTACIÓN. Roma. Disponible en: www.fao.org/3/ai0480s.pdf.

7. Goering RV. Pulsed field gel electrophoresis: a review of application and interpretation in the molecular epidemiology of infectious disease. Infect Genet Evol. 2010 Oct;10(7):866-75. Disponible en: doi: 10.1016/j.meegid.2010.07.023. Epub 2010 Aug 6. Review. PMID: 20692376

8. Muñoz N, Realpe M, Castañeda E, Agudelo C. Caracterización por electroforesis de campo pulsado de aislamientos de Salmonella Typhimurium recuperados en el programa de vigilancia de enfermedad diarreica aguda en Colombia, 1997-2004. Biomédica 2006;26:397-407. Disponible en:

http://www.redalyc.org/articulo.oa?id $=843$ 32609

9. Rios M, Araya P, Fernandez A, Tognarelli J, Hormozabal JC. Fernández J. Subtipificación molecular de Salmonella entérica serotipo Enteritidis en el período post epidémico. Chile. Rev Méd Chile 2009;137:71-7. Disponible en: http://dx.doi.org/10.4067/S003498872009000100010.

10. Diaz M, Diaz P, Rodriguez E, Montaño $L$, Medina M, Realpe ME et al. Caracterización fenotípica y genotípica de Salmonella Typhimurium variante 5- asociada a un brote de enfermedad transmitida por alimentos en el municipio de Paz de Río, Boyacá, 2010. IATREIA2014; 27(1):23-30. Disponible https://aprendeenlinea udea edu co/revist as/index.php/.../14327.

11. Campos J, Pichel M, Vaz T, Tavechio A, Fernandes S. Building PulseNet Latin America and Caribbean Salmonella regional database: First conclusions of genetic subtypes of S. Typhi, S. Typhimurium and $S$. Enteritidis circulating in six countries of the region. Food Research International (2011). Disponible en: doi: 10.1016/j.foodres.2011.10.020.

12. Zamudio M, Meza A, Bailon H, Martinez J, Campos J. Experiencias en la vigilancia epidemiológica de agentes patógenos transmitidos por alimentos a través de electroforesis en campo pulsado (pfge) en el Perú. Rev Peru Med Exp Salud Publica. 2011;28(1):128-35. Disponible en: hppt://www.sisbib.unmsm.edu.pe/bvrevist as/medicina.../v28.../a21v28n1.pdf

13. Vélez $N$, Díaz $P$, Rodríguez $C$, Bautista $A$, Montaño L, Realpe ME. Caracterización molecular de aislamientos de Shigella sonnei recuperados en el programa de vigilancia por el laboratorio de la enfermedad diarreica aguda en Colombia. Biomédica 2015;35:395-406. Disponible en: doi: http://dx.doi.org/10.7705/biomedica.v35i3 .2622

14. Ruiz A, Torres M, Aznar J. Molecular epidemiology and antimicrobial susceptibility of Campylobacter coli clinical isolates. Enferm Infecc Microbiol Clin. 2014. Disponible en: http://dx.doi.org/10.1016/j.eimc.2014.02. 012

15. Kalender $H$, Kilic A. Molecular characterisation of Shiga toxin-producing Escherichia coliO157:H7 isolates from cattle in eastern Turkey. Veterinarni Medicina, 61, 2016 (12): 663-668. Disponible en: doi: 10.17221/45/2016VETMED

16. Oderiz S, Leotta G, Galli L. Detección y caracterización de Escherichia coli productor de toxina Shiga en niños atendidos en un hospital pediátrico interzonal de la ciudad de La Plata. Rev Argent Microbiol. 2018. Disponible en: https://doi.org/10.1016/j.ram.2017.08.008 
17. Ribot EM, Fair MA, Gautom R, Cameron DN, Hunter SB, Swaminathan B et al. Standardization of pulsed-field gel electrophoresis protocols for the subtyping of Escherichia coli O157:H7, Salmonella, and Shigella for PulseNet. Foodborne Pathog Dis. 2006;3(1):59-67. Disponible en:

https://www.ncbi.nlm.nih.gov/pubmed/16 602980

18. Hunter $S$, Vauterin $P$, Lambert-Fair $M$, Van $M$, Kubota K, Graves $L$, et al. Establishment of a universal size standard strain for use with the PulseNet standardized pulsed-field gel electrophoresis protocols: Converting the national databases to the new size standard. Journal of Clinical Microbiology, 2005;43:1045-50. Disponible en: https://www.ncbi.nlm.nih.gov/pubmed/15 750058.

19. Gerner P, Hise K, Kincaid J, Hunter S, Rolando S, Hyytia-Trees, E et al. PulseNet USA: a five-year update. Foodborne Pathog Dis. 2006 Spring; 3(1):9-19. Disponible en: https://www.ncbi.nlm.nih.gov/pubmed/16 602975

20. Tenover FC, Arbeit RD, Goering RV, Mickelsen PA, Murray BE, Persing DH et al. Interpreting chromosomal DNA restriction patterns produced by Pulsed-Field Gel
Electrophoresis: criteria for bacterial strain typing.. Journal of Clinical Microbiology 1995; 33: 2233-2239. Disponible en: https://www.ncbi.nlm.nih.gov/pubmed/74 94007

21. Organización Panamericana de la Salud. Salud en la Américas 2012. Panorama regional y perfiles de país. Washington, DC. 2012; Volumen de países: 567-583. Disponible en: http://iris.paho.org/xmlui/handle/1234567 89/3272.

22. Reyes N, Talavera M, Varela J, Barba J, Gutiérrez A, Alonso-Fresan U. Prevalencia y resistencia a antibióticos de Escherichia coliO157:H7 aislada de canales de bovinos sacrificados en rastros del altiplano central Mexicano. Rev Mex Cienc Pecu 2013;4(2):235-242. Disponible en: http://www.scielo.org.mx/scielo.php?script =sci_arttext\&pid =S200711242013000200009 \&lng $=$ es.

23. Weiler $N$, Orrego $M$, Alvarez $M$, Huber $C$. Detección molecular de Escherichia coli diarreogénicas en pacientes pediátricos con síndrome diarreico agudo. Paraguay. Mem. Inst. Investig. Cienc. Salud. 2017;15(1): 16-21. Disponible en: http://scielo.iics.una.py/pdf/iics/v15n1/18 12-9528-iics-15-01-00016.pdf 